\title{
Dynamical model of the dielectric screening of conjugated polymers
}

\author{
William Barford ${ }^{1 *}$, Robert J. Bursill ${ }^{2}$ and David Yaron ${ }^{3}$ \\ ${ }^{1}$ Department of Physics and Astronomy, \\ University of Sheffield, Sheffield, S3 7RH, United Kingdom. \\ ${ }^{2}$ School of Physics, University of New South Wales, Sydney, NSW 2052, Australia. \\ ${ }^{3}$ Department of Chemistry, Carnegie Mellon University, Pittsburgh, PA 15213, U.S.A.
}

\begin{abstract}
A dynamical model of the dielectric screening of conjugated polymers is introduced and solved using the density matrix renormalization group method. The model consists of a line of quantized dipoles interacting with a polymer chain. The polymer is modelled by the Pariser-Parr-Pople (P-PP) model. It is found that: (1) Compared to isolated, unscreened single chains, the screened $1^{1} B_{u}^{-}$ exciton binding energy is typically reduced by ca. $1 \mathrm{eV}$ to just over $1 \mathrm{eV}$. (2) Covalent (magnon and bi-magnon) states are very weakly screened compared to ionic (exciton) states. (3) Screening of the $1^{1} B_{u}^{-}$exciton is closer to the dispersion than solvation limit.
\end{abstract}

PACS numbers: 78.67.-n, 77.22.-d 


\section{INTRODUCTION}

The intra-chain excitations of a conjugated polymer in the solid state are often strongly screened by the environment of the other polymers. This screening causes significant reductions of the ionic single-chain excitation energies and charge gaps, and thus single-chain calculations are expected to considerably over-estimate the actual excitation energies of polymers in the solid state. In particular, the energy of a point charge is typically reduced by approximately $1 \mathrm{eV}$, while the excitation energy of the lowest dipole allowed exciton is reduced by approximately $0.5 \mathrm{eV}$, implying a deviation of approximately $1.5 \mathrm{eV}$ in the singlechain calculated exciton binding energy in comparison to the solid state measurements. $\underline{1}^{2}$

It is useful to consider two limiting cases for the interaction between the electron-hole pair and the polarization of the dielectric medium, namely solvation-like versus dispersion-like interactions. By solvation, we mean a polarization that develops around the electron and hole and follows the motion of these charged species (that is, the dielectric is fast compared to the electron-hole motion). In this case, the effects of the polarization can be absorbed into a static screened electron-hole Coulomb interaction. By dispersion, we mean the interaction between fluctuating dipoles, such as may arise between the fluctuating dipole of the bound electron-hole pair in an exciton and the dipoles of the dielectric medium. The interactions become dispersion-like when the time scale of the electron-hole motion is comparable to or less than the time scale of the dielectric response (that is, the electron-hole motion is fast compared to the dielectric). In this case, the dielectric polarization does not identically follow the electron-hole motion, but rather fluctuates in a manner that is correlated with the motion of the electron-hole pair. Now, the effects of the polarization may be modelled by a dynamically screened electron-hole Coulomb interaction.

The nature of the screening thus depends on the relative time scale of the electron-hole motion, which is approximately inversely proportional to the exciton binding energy $\mathrm{y}^{\underline{3}}$, versus the time scale of the dielectric polarization, which is inversely proportional to the optical gap of the dielectric medium. In inorganic semiconductors, the exciton binding energy is one to two orders of magnitude smaller than the optical gap. The polarization is then much faster than the electron-hole motion, such that it can follow the electron-hole motion and effectively screen the Coulomb interaction. ${ }^{\underline{4}}$ In organic semiconductors, the exciton binding energy is comparable to the optical gap and we expect the interaction between the exciton 
and the dielectric polarization to have a dispersion-like character. In this regime, there is no a priori reason to suppose that a static dielectric response function will correctly describe the relevant physics ${ }^{2}$.

In this paper we present a dynamic model of the dielectric - making no assumptions about time scales - with which we attempt to understand the screening of the key excited states

of conjugated polymers. We consider the rôle of lengths scales (namely, the electron-hole separation versus the length scales in the monopole-dipole interaction), time scales (namely, exciton excitation energy versus binding energy), and ionicity versus covalency in the excited states. The correlated electron dynamics within a single chain are modelled by the PariserParr-Pople (P-P-P) model, while the dielectric is treated as a linear chain of quantized dipoles. This model is solved essentially exactly via the density matrix renormalization group (DMRG) method..$^{\underline{5}}$

The plan of this paper is as follows: in the next section the model is introduced and parameterized. In the following section we discuss our results, and finally conclude.

\section{DYNAMIC DIELECTRIC MODEL}

The model is designed to represent the dielectric response of an array of conjugated polymers parallel to the solute (or test) chain. The dielectric function, $\epsilon(\omega)$, of an assembly of polymers is $\frac{6}{}$,

$$
\epsilon(\omega)=1+\frac{N q^{2}}{\epsilon_{0} m} \sum_{n} \frac{f_{n}}{\omega_{n}^{2}-\omega^{2}},
$$

where $\omega_{n}$ and $f_{n}$ are the frequency and oscillator strength for the $n$th transition, $q$ and $m$ are the electronic charge and mass, $\epsilon_{0}$ is the dielectric constant of free-space, and $N$ is the number of polymers per unit volume. In this paper we consider a 'two-state' model, namely we only consider excitations of the dielectric from the ground state to the first dipole-allowed state (e.g. the $1^{1} B_{u}$ state in centro-symmetric polymers). Then, using the oscillator sum rule,

$$
\sum_{n} f_{n}=1
$$

where,

$$
f_{n}=\frac{2 m \omega_{n}\langle G S|\hat{\mu}| n\rangle^{2}}{q^{2} \hbar}
$$




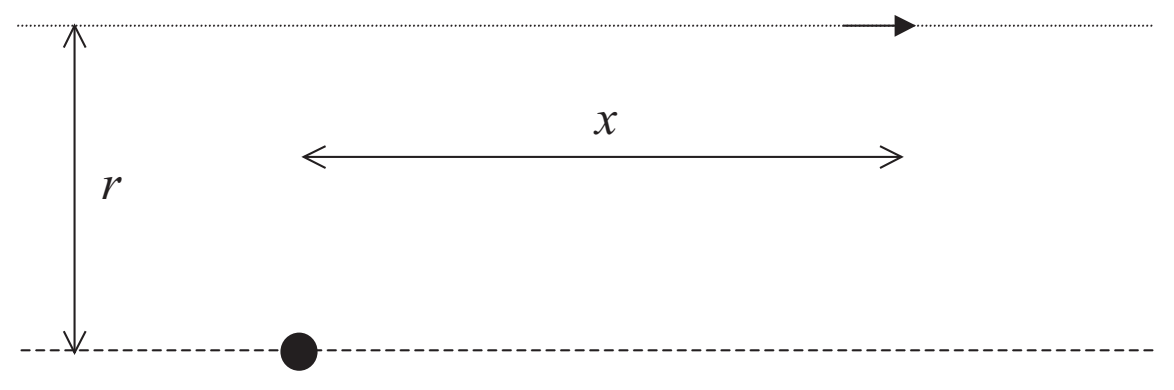

FIG. 1: The polymer chain (dashed line), with a monopole at $x_{i}$ (circle) and the line of longitudinal dipoles (dotted line), with a dipole at $x_{j}$ (arrow).

we have

$$
\epsilon(\omega)=1+\frac{N}{\epsilon_{0}} \frac{2\langle\hat{\mu}\rangle^{2} \omega_{0}}{\hbar\left(\omega_{0}^{2}-\omega^{2}\right)} .
$$

In this expression, $\langle\hat{\mu}\rangle$ and $\omega_{0}$ are the transition dipole moment and frequency of the excited state. $\hbar \omega_{0}$ corresponds to the optical gap of the polymers. We note that for $\omega>>\omega_{0}$ Eq. (41) becomes,

$$
\epsilon(\omega)=1-\frac{\omega_{p}^{2}}{\omega^{2}}
$$

where $\omega_{p}^{2}=N q^{2} / \epsilon_{0} m$ is the plasma frequency. This frequency is typically $10-20 \mathrm{eVs}$ much larger than the optical gap or the energy scale of the electron-hole motion - so this limit need not concern us here.

Since the dipole moment of the chain is parallel to the chain axis, we consider a line of longitudinal dipoles, each oscillating with a characteristic frequency, $\omega_{0} \stackrel{\underline{7}}{\text { The dipole at }} x_{j}$ interacts with a point-charge on the solute chain at $x_{i}$ via the monopole-dipole interaction,

$$
U_{s}(x, r)=\frac{q x \mu}{4 \pi \epsilon_{0}\left(x^{2}+r^{2}\right)^{3 / 2}},
$$

where $\mu$ is the dipole moment, $x=\left(x_{j}-x_{i}\right)$ and $r$ is the normal distance of the line of dipoles from the chain, as illustrated in Fig. 1.

A realistic model of the dielectric would consist of a three-dimensional array of lines of dipoles surrounding the solute chain. However, such a model would not be easily soluble by the DMRG method. Instead, we consider a one-dimensional model and derive an effective interaction between the monopoles and the three-dimensional array of dipoles by integrating over a cylinder with inner and outer radii of $r_{1}$ and $r_{2}$, respectively:

$$
U\left(x ; r_{1}, r_{2}\right)=\int_{r_{1}}^{r_{2}} \rho 2 \pi a r U_{s}(x, r) \mathrm{d} r
$$




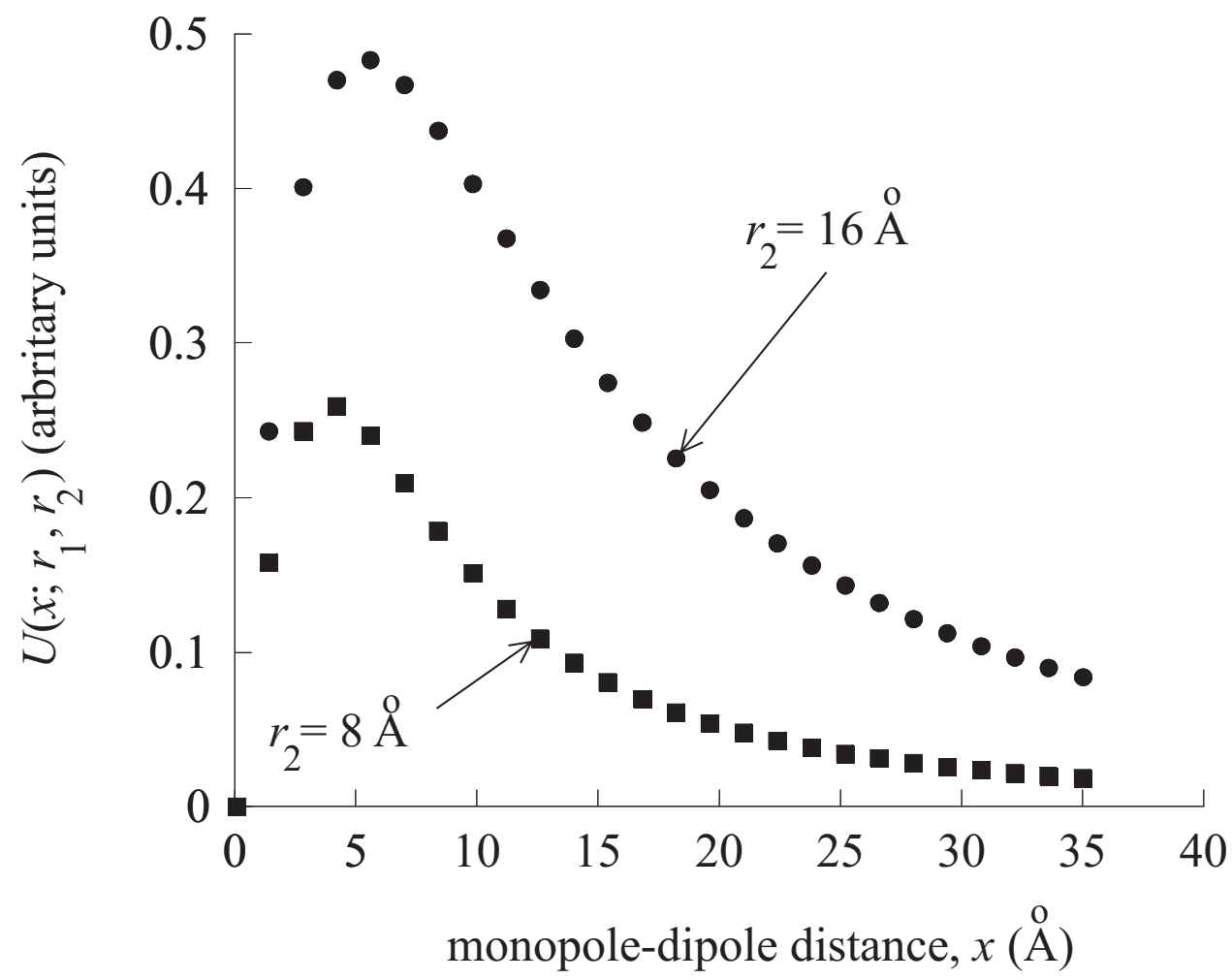

FIG. 2: The integrated monopole-dipole interaction, $U\left(x ; r_{1}, r_{2}\right)$, for $r_{1}=4 \AA$, with $r_{2}=8 \AA$ (squares) and $r_{2}=16 \stackrel{\AA}{\AA}$ (circles).

$$
=\left(\frac{q}{4 \pi \epsilon_{0}}\right)\left(\frac{2 \pi a}{r_{1}^{2}}\right)\left(\rho r_{1}^{2} \mu\right)\left[\sin \left(\tan ^{-1}\left(\frac{x}{r_{1}}\right)\right)-\sin \left(\tan ^{-1}\left(\frac{x}{r_{2}}\right)\right)\right] \text {. }
$$

Here, $\rho$ is the number density of dipoles and $a$ is the linear separation (for convenience taken to be the separation between the sites on the chain). The purpose of this integration procedure is that it performs a type of average over the distances between the line dipoles and the solute chain. However, $r_{1}$ is still the effective length scale in the interaction, as shown in Fig. 2, which shows $U\left(x ; r_{1}, r_{2}\right)$ for different $r_{2}$ for fixed $r_{1}$. For both $r_{2}=8 \AA$ and $16 \stackrel{\circ}{A} U\left(x ; r_{1}, r_{2}\right)$ is strongly peaked at approximately the value of $r_{1}$. Thus, using a single line dipole a distance $r_{1}$ away would give very similar results to the cylinder of inner radius $r_{1}$.

The Hamiltonian is thus,

$$
H=H_{e}+H_{d}+H_{e-d},
$$


where,

$H_{e}=-\sum_{i \sigma} t_{i}\left(c_{i \sigma}^{\dagger} c_{i+1 \sigma}+c_{i+1 \sigma}^{\dagger} c_{i \sigma}\right)+U \sum_{i}\left(\hat{N}_{i \uparrow}-\frac{1}{2}\right)\left(\hat{N}_{i \downarrow}-\frac{1}{2}\right)+\frac{1}{2} \sum_{i \neq j} V_{i j}\left(\hat{N}_{i}-1\right)\left(\hat{N}_{j}-1\right)(9)$

is the P-P-P model, describing the interacting electronic degrees of freedom. $c_{i \sigma}^{\dagger}$ creates an electron with spin $\sigma$ in the $\pi$-orbital on site $i$ and $\hat{N}_{i}=\sum_{\sigma} c_{i \sigma}^{\dagger} c_{i \sigma} . V_{i j}$ is the Ohno-Coulomb repulsion,

$$
V_{i j}=\frac{U}{\sqrt{1+\epsilon \beta r_{i j}^{2}}}
$$

where $\epsilon$ is the dielectric constant (generally taken to be 1 ), the bond lengths are in $\AA$ and $\beta=(U / 14.397)^{2}$. The bond lengths, $a$, used in the evaluation of $V_{i j}$ are $1.4 \AA$, and the bond angle is $180^{\circ}$. The transfer integral is $t_{i}=t\left(1+(-1)^{i} \delta\right)$, where $\delta$ is the bond dimerization parameter.

The Hamiltonian,

$$
H_{d}=\sum_{j} \hbar \omega_{0} b_{j}^{\dagger} b_{j}
$$

describes the oscillating dipoles. $b_{j}^{\dagger}$ is a boson operator creating a quantum of energy $\hbar \omega_{0}$ in a linear harmonic oscillator located at $x_{j}$. This term describes the non-interacting self-energy of the oscillating dipoles. However, in this model we neglect the dipole-dipole interactions, as in one dimension this induces an unphysical spontaneous dipole moment. Thus, the classical limit (defined as $\omega_{0} \rightarrow 0$ ) is not completely described by this model.

Using Eq. (71), the monopole-dipole Hamiltonian is,

$$
H_{e-d}=\sum_{i j}\left(\frac{q^{2}}{4 \pi \epsilon_{0}}\right)\left(\frac{2 \pi a}{r_{1}^{2}}\right)\left[\sin \left(\tan ^{-1}\left(\frac{x}{r_{1}}\right)\right)-\sin \left(\tan ^{-1}\left(\frac{x}{r_{2}}\right)\right)\right]\left(\hat{N}_{i}-1\right) \lambda \frac{\left(b_{j}^{\dagger}+b_{j}\right)}{2},
$$

where we define the dimensionless monopole-dipole coupling constant, $\lambda$, as,

$$
\lambda=\rho r_{1}^{2}\langle\hat{d}\rangle
$$

and we have used

$$
\hat{\mu}=q\langle\hat{d}\rangle \frac{\left(b_{j}^{\dagger}+b_{j}\right)}{2} .
$$

$q\langle\hat{d}\rangle$ is the transition dipole moment of the solvent chains associated with the $1^{1} B_{u}^{-}$exciton ${ }^{8}$ and $\left(b_{j}^{\dagger}+b_{j}\right) / 2$ is the dimensionless operator corresponding to the displacement of the oscillator. 
In the absence of electron-electron interactions and for only local monopole-dipole interactions the Hamiltonian, $H$, is the Holstein model, which is widely used to study chargedensity-wave phenomena in molecular solids. The monopole-dipole interactions imply that $H$ possesses a special type of particle-hole symmetry. In particular, $H$ is invariant under the simultaneous particle-hole transformation of $\underline{9}$

$$
c_{i \sigma}^{\dagger} \rightarrow(-1)^{i} c_{i \bar{\sigma}}
$$

and a reversal of parity in the boson operator,

$$
b_{j}^{\dagger} \rightarrow-b_{j}^{\dagger} .
$$

$\hbar \omega_{0}$ is the excitation energy of the $1^{1} B_{u}$ exciton and $q\langle\hat{d}\rangle$ is its corresponding transition dipole moment. These are determined by solving the single chain P-P-P model in the absence of monopole-dipole interactions. $\rho$ is the only adjustable parameter in the model, and is adjusted to reproduce the solvation energy of a point charge found by Yaron and Moore $e^{2}$ for particular values of the P-P-P parameters, namely, $t, U$ and $\delta$. Having fixed $\rho$ for one set of P-P-P parameters the model can be transferred to another set of P-P-P parameters, by recalculating the unscreened values of $\hbar \omega_{0}$ and $\langle\hat{d}\rangle$, and the solvation of states as a function of these parameters can be monitored.

\section{A. Parametrization of the model}

Moore and Yaron ${ }^{2}$ studied solvation effects in polyacetylene by surrounding a central solute chain by increasing numbers of solvent chains, arranged as in the crystal structure of ref $\stackrel{10}{ }$, and extrapolating to an infinite system. The central chain was treated with a PP-P model with parameters, $t=2.4045 \mathrm{eV}, \delta=0.0734, U=11.13 \mathrm{eV}$ (corresponding to $t_{d}=2.581 \mathrm{eV}$ and $\left.t_{s}=2.228 \mathrm{eV}\right)$ and the solvent chains were treated in an independentelectron approximation. Coulomb interactions were included between all chains. Replacing the central solute chain with a point charge led to an estimate of about $1 \mathrm{eV}$ for the solvation energy of a point charge. In the assumption of an infinitely fast dielectric, where the solvent polarization is equilibrated to the instantaneous position of the electron and hole, the solvation energy of a well separated electron and hole is about $1.9 \mathrm{eV}$. Using a model that approximates the dynamic response of the dielectric, the free electron and hole become 
TABLE I: Unscreened energies, the transition moment, $\langle\hat{d}\rangle$, and the dipole 'polarizability', $\alpha=$ $\frac{\langle\hat{d}\rangle^{2}}{E\left(1^{1} B_{u}^{-}\right)}$of a 50 -site polymer chain modelled by the P-P-P model. The energies are in eVs.

\begin{tabular}{|c|c|c|c|c|c|c|c|}
\hline$t$ & $U$ & $\delta$ & $\mathrm{E}\left(1^{1} B_{u}^{-}\right) 1^{1} B_{u}^{-}$ & binding energy & $\mathrm{E}\left(2^{1} A_{g}^{+}\right)$ & $\mathrm{E}\left(1^{3} B_{u}^{+}\right)$ & $\langle\hat{d}\rangle \AA$ \\
\hline 2.4045 & 11.13 & 0.0734 & 2.85 & 2.29 & 2.31 & 1.28 & $8.72 \quad 26.7$ \\
\hline
\end{tabular}

dressed by the dielectric response of the solvent chains to form polarons and the solvation energy drops to about $1.5 \mathrm{eV}$. Using the ab initio DFT-GWA-BSE method, Bobbert and co-workers calculated a point charge screening in polythiophene of $0.55 \mathrm{eV}$, i.e. 1.1 for the charge gap ${ }^{11}$.

Notice that for the parameter values used by Moore and Yaron the P-P-P model is strongly correlated ${ }^{12}$. The triplet, $1^{3} B_{u}^{+}$, state is more properly described as a magnon than the $n=1$ Mott-Wannier triplet exciton. Similarly, the $2^{1} A_{g}^{+}$state is not the $n=2$ MottWannier singlet exciton, but a bi-magnon, whose energy lies below the $n=1$ singlet exciton, namely the $1^{1} B_{u}^{-}$state ${ }^{13.14}$ The unscreened energies of a 50 site chain are shown in Table I.

We solve Eq. (8) by the DMRG method ${ }^{5}$ using a program well-tested on similar electronboson models $\frac{15}{}$. Since the boson energy scale, $\hbar \omega_{0}$, is comparatively large compared to the electronic excitation energies, we found that only one boson per site is required for the excitation energies to converge to within $0.01 \mathrm{eV}$. The number of superblock states was typically $\sim 100,000$ and one finite-lattice sweep at the target chain size was always performed to ensure convergence.

Taking $r_{1}=4 \stackrel{\circ}{A}, r_{2}=16 \stackrel{\circ}{A}$ and $\lambda=0.14$, we obtain a screening energy of $0.81 \mathrm{eV}$ for the point charge. This is intermediate between the values of Bobbert ${ }^{11}$ and Moore-Yaron ${ }^{2}$ and is close to the charged-polaron result of 0.88 for 50 sites. $^{2}$ The distance dependence of the charge-charge screening arising from the dielectric model of Eq. (77) is similar to that obtained from the explicit solvent model of Moore and Yaron ${ }^{2}$, suggesting that the longitudinal dipoles provide a reasonable description of the dielectric medium.

The excitation energy of the $1^{1} B_{u}^{-}$exciton is reduced by $0.69 \mathrm{eV}$, which is roughly twice as large as the Yaron-Moore result ${ }^{2}$, and much larger than the DFT-GWA-BSE calculation, which predicts a negligible screening of the exciton ${ }^{11}$. The binding energy is therefore reduced from $2.3 \mathrm{eV}$ to $1.4 \mathrm{eV}$. As already noted, for these parameter ranges the $1^{3} B_{u}^{+}$state is a magnon and the $2^{1} A_{g}^{+}$state is a bi-magnon. Both states are therefore strongly covalent with 
TABLE II: Screened energies, E, screening energies, S, and the screened $1^{1} B_{u}^{-}$binding energy, $\mathrm{BE}$, of a 50 -site polymer chain. The charge gap is denoted by $2 \Delta$. The energies are in eVs.

\begin{tabular}{|c|c|c|c|c|c|c|c|c|c|c|c|c|}
\hline$t$ & $U$ & $\delta$ & $\lambda$ & $\mathrm{E}\left(1^{1} B_{u}^{-}\right)$ & $\mathrm{S}\left(1^{1} B_{u}^{-}\right)$ & $\mathrm{E}\left(2^{1} A_{g}^{+}\right)$ & $\mathrm{S}\left(2^{1} A_{g}^{+}\right)$ & $\mathrm{E}\left(1^{3} B_{u}^{+}\right)$ & $\mathrm{S}\left(1^{3} B_{u}^{+}\right)$ & $2 \Delta$ & $\mathrm{S}(2 \Delta)$ & $\mathrm{BE}$ \\
\hline 2.4045 & 11.13 & 0.0734 & 0.14 & 2.16 & 0.69 & 2.22 & 0.09 & 1.25 & 0.03 & 3.53 & 1.62 & 1.37 \\
\hline
\end{tabular}

very little ionicity and thus there is very little screening. This affect can be traced back to the monopole-dipole interaction, Eq. (12), which shows that the electric dipole couples to charge density (or ionic) fluctuations in the wavefunction. These results are summarized in Table II.

\section{RESULTS}

Having parameterized the model, we now investigate other parameter ranges. $\lambda$ is found using Eq. (13) for fixed $\rho$ and $r_{1}$ with the recalculated value of $\langle\hat{d}\rangle$. First, we consider $U=10 \mathrm{eV}, t=2.5 \mathrm{eV}$ and $\delta=0.1$, as this corresponds to the optimal parameter set used by Barford and Bursill in their study of trans-polyacetylene ${ }^{16}$. The single chain P-P-P excitation energies are shown in Table III. Notice that, unlike the case for the original PP-P model parametrization, the $2^{1} A_{g}^{+}$state lies higher in energy than the $1^{1} B_{u}^{-}$state. The screened energies are shown in Table IV, where we see that the charge gap is solvated by ca. $2.0 \mathrm{eV}$, and the $1^{1} B_{u}^{-}$and $2^{1} A_{g}^{+}$states are screened by $0.80 \mathrm{eV}$ and $0.75 \mathrm{eV}$, respectively. The binding energy of the $1^{1} B_{u}^{-}$exciton is reduced from 2.3 to $1.1 \mathrm{eV}$.

We next investigate the rôle played by the strength of the Coulomb interactions, particularly on the screening of the $2^{1} A_{g}^{+}$state, which evolves from a bi-magnon to the $n=2$ Mott-Wannier singlet exciton, and the triplet, $1^{3} B_{u}^{+}$, state, which evolves from a magnon to the $n=1$ Mott-Wannier triplet exciton, as $U$ is reduced ${ }^{13,14}$. We take a value for $\delta$ of 0.2 as this maps the linear chain band structure onto the band structure of poly(p-phenylene). Table IV confirms the expected trend that as the ionicity of the $2^{1} A_{g}^{+}$and $1^{3} B_{u}^{+}$states increases relative to their covalency their screening, relative to the ionic $1^{1} B_{u}^{-}$state, increases.

Length scales also play a rôle in the screening of the excited states and the charge gap. As the separation between a polymer and a line of dipoles, $r$, is increased the typical distance 
TABLE III: Unscreened energies, the transition moment, $\langle\hat{d}\rangle$, and the dipole 'polarizability', $\alpha=\frac{\left\langle\hat{d}^{2}\right.}{E\left(1^{1} B_{u}^{-}\right)}$of a 50 -site polymer chain modelled by the P-P-P model. The energies are in eVs.

\begin{tabular}{|c|c|c|c|c|c|c|c|c|}
\hline$t$ & $U$ & $\delta$ & $\mathrm{E}\left(1^{1} B_{u}^{-}\right) 1^{1} B_{u}^{-}$ & binding energy & $\mathrm{E}\left(2^{1} A_{g}^{+}\right)$ & $\mathrm{E}\left(1^{3} B_{u}^{+}\right)$ & $\langle\hat{d}\rangle \AA$ & $\alpha$ \\
\hline 2.5 & 10 & 0.1 & 2.67 & 2.32 & 2.95 & 1.69 & 9.41 & 33.2 \\
\hline 2.5 & 10 & 0.2 & 3.69 & 3.05 & 4.69 & 2.76 & 8.15 & 18.0 \\
\hline 2.5 & 8 & 0.2 & 3.20 & 2.76 & 4.42 & 2.82 & 8.81 & 24.3 \\
\hline 2.5 & 6 & 0.2 & 2.93 & 2.42 & 4.10 & 2.79 & 9.14 & 28.5 \\
\hline
\end{tabular}

TABLE IV: Screened energies, E, screening energies, S, and the screened $1^{1} B_{u}^{-}$binding energy, $\mathrm{BE}$, of a 50 -site polymer chain. The charge gap is denoted by $2 \Delta$. The energies are in eVs.

\begin{tabular}{ccccccccccccc}
\hline \hline$t$ & $U$ & $\delta$ & $\lambda$ & $\mathrm{E}\left(1^{1} B_{u}^{-}\right)$ & $\mathrm{S}\left(1^{1} B_{u}^{-}\right)$ & $\mathrm{E}\left(2^{1} A_{g}^{+}\right)$ & $\mathrm{S}\left(2^{1} A_{g}^{+}\right)$ & $\mathrm{E}\left(1^{3} B_{u}^{+}\right)$ & $\mathrm{S}\left(1^{3} B_{u}^{+}\right)$ & $2 \Delta$ & $\mathrm{S}(2 \Delta)$ & $\mathrm{BE}$ \\
\hline 2.5 & 10 & 0.1 & 0.151 & 1.87 & 0.80 & 2.20 & 0.75 & 1.59 & 0.10 & 3.00 & 1.99 & 1.13 \\
2.5 & 10 & 0.2 & 0.131 & 3.22 & 0.47 & 4.34 & 0.35 & 2.73 & 0.03 & 5.43 & 1.31 & 2.21 \\
2.5 & 8 & 0.2 & 0.141 & 2.60 & 0.60 & 3.45 & 0.97 & 2.71 & 0.11 & 4.26 & 1.70 & 1.66 \\
2.5 & 6 & 0.2 & 0.147 & 2.19 & 0.74 & 2.48 & 1.62 & 2.40 & 0.39 & 3.26 & 2.09 & 1.07 \\
\hline \hline
\end{tabular}

in the monopole-dipole interaction increases. When this interaction distance is larger than the particle-hole separation, the charge character of the exciton approaches that of a point dipole. For the line dipole used here, the ratio of the screening energy of the $1^{1} B_{u}^{-}$state versus the charge gap is 0.6 when $r=4 \stackrel{\AA}{A}$ and reduces to a roughly constant value of 0.4

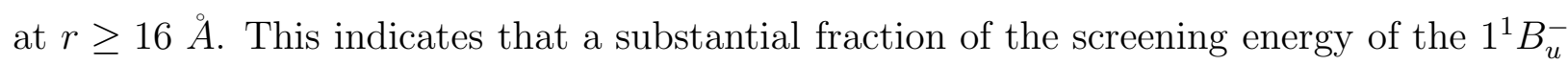
exciton is from dipole-dipole interactions.

\section{CONCLUSIONS}

In this paper we introduced a dynamical model of a dielectric to investigate the screening of intra-chain excitations by the environment. This model consisted of a linear array of quantized longitudinal dipoles interacting with a conjugated polymer, represented by the Pariser-Parr-Pople model. The dipole parameters are adjusted to provide a realistic model of the dielectric. The model reproduces the expected solvation energy of a point charge in these 
materials and sets the frequency of the dielectric response to the optical gap of the material. The model is one-dimensional and is solved by the density matrix renormalization group method. The one-dimensionality of the model does mean that it is impossible to include dipole-dipole interactions, and thus the model misses some of the physical characteristics of real dielectric materials.

We make the following conclusions from our results. (1) The $1^{1} B_{u}^{-}$exciton binding energy is typically reduced by ca. $1 \mathrm{eV}$ to just over $1 \mathrm{eV}$. Thus, this relatively simple onedimensional model of the screening of conjugated polymers by the environment predicts $1^{1} B_{u}^{-}$binding energies rather close to the experimentally accepted solid state values of ca. $1 \mathrm{eV}$. (2) Covalent (magnon and bi-magnon) states are very weakly screened compared to ionic (exciton) states. (3) Screening of the $1^{1} B_{u}^{-}$exciton is closer to the dispersion than solvation limit.

\section{Acknowledgments}

We thank Eric E. Moore for useful discussions. This work was supported by the U. K. Engineering and Physical Sciences Research Council (GR/R03921). R. J. B. is supported by the Australian Research Council and the J G Russell Foundation. D. Y. J. is supported by the US National Science Foundation (0316759).

* E.mail address: W.Barford@sheffield.ac.uk

1 E. Moore, B. Gherman and D. Yaron, J. Chem. Phys. 106, 4216 (1997).

2 E. E. Moore and D. Yaron, J. Chem. Phys. 109, 6147 (1998).

3 Or more correctly, the time-scale for the electron-hole motion is inversely proportional to the energy gap to the next dipole-connected excited state (e. g. $\left(E\left(m A_{g}\right)-E\left(1 B_{u}\right)\right)$ for centrosymmetric polymers).

4 R. Knox, Theory of Excitons, Solid State Physics: Supplement 5, Academic Press, New York, (1963).

5 S. R. White, Phys. Rev. Lett. 69, 2863 (1992); Density Matrix Renormalization, edited by I. Peschel, X. Wang, M. Kaulke and K. Hallberg (Springer, Berlin 1999). 
6 See, Principles of the Theory of Solids, Z. M. Ziman (2nd ed.) Cambridge University Press, Cambridge (1972).

7 In principal, the model could be extended to include transverse dipoles by including an additional flavor of boson in the model described in Eq. (8).

8 The transition moment operator is defined as $\hat{d}=\sum_{i}\left(\hat{N}_{i}-1\right) x_{i}$. Thus, the transition moment associated with the $1^{1} B_{u}^{-}$state, $\langle\hat{d}\rangle$, is $\left\langle 1^{1} B_{u}^{-}|\hat{d}| 1^{1} A_{g}^{+}\right\rangle$.

9 One consequence of this symmetry is that the electronic ground state is associated with a manifold of 'vibrational' states, with a spacing $\hbar \omega_{0}$ and alternating between ${ }^{1} A_{g}^{+}$and ${ }^{1} B_{u}^{-}$ symmetry. Thus, the $1^{1} B_{u}^{-}$state does not necessarily correspond to the lowest 'vibrational' state of the excited exciton. We check that it does by calculating its dipole moment to the $1^{1} A_{g}^{+}$ state.

10 C. R. Fincher, C. E. Chen, A. J. Heeger, A. G. MacDiarmid and J. B. Hastings, Phys. Rev. Lett. 48, 100 (1982).

11 J.-W. van der Horst, P. Bobbert, M. A. J. Michels, G. Brocks and P. J. Kelly, Phys. Rev. Lett. 83, 4413 (1999).

12 I. Ohmine, M. Karplus, and K. Schulten, J. Chem. Phys., 68, 2298 (1978).

13 W. Barford, Phys. Rev. B 65, 205118 (2002).

14 W. Barford, R. J. Bursill and R. W. Smith, Phys. Rev. B 66, 115205 (2002).

15 W. Barford, R. J. Bursill and M. Yu Lavrentiev, Phys. Rev. B 65, 75107 (2002).

16 W. Barford, R. J. Bursill and M. Yu Lavrentiev, Phys. Rev. B 63, 195108 (2001). 